\title{
Verification of the Dynamic Modeling of 2-R Robot Actuated by (N) Equally Spaced Planet-Gears by Using SolidWorks and MATLAB/SIMULINK
}

\author{
Brahim FERNINI \\ Mustapha Temmar \\ University Center of Tissemsilt \\ Laboratory of Structures Blida1 University, Algeria \\ fernini.brahim12@gmail.com \\ temmarmustapha@yahoo.fr \\ Yoshihiro KA \\ Department of Mechanical Engineering \\ Tokai University, Hiratsuka, Kanagawa 259-1292, Japan \\ kai@keyaki.cc.u-tokai.ac.jp \\ Muhamad M. Noor \\ Faculty of Mechanical Engineering \\ Universiti Malaysia Pahang (UMP), Malaysia \\ muhamad@ump.edu.my \\ Received (19 June 2018) \\ Revised (2 July 2018) \\ Accepted (15 August 2018)
}

Industrial robots often use planetary gear system to have high joint torques; therefore, the influence of the rotary inertia of the number of the equally spaced planet-gears on the dynamical behavior of the robot is very important. The main objective of this paper is to develop the dynamic modeling of robot actuated by ( $n$ ) equally spaced planet-gears in the case where the planet-carrier is fixed, no closed solution has been reported for this dynamic modeling, and to compare between the dynamic behavior of robot actuated by $(n+1)$ and $(n)$ equally spaced planet-gears for a same trajectory planning. The authors derive the explicit dynamic model for an elbow down of 2-R manipulator holding an external mass. Finally, the obtained simulation results by using Matlab/Simulink of the dynamic modeling are verified by modeling the same robot and using an advanced simulation via SolidWorks (2014).

Keywords: (n) equally spaced Planet-Gears, kinetic energy, joint torque. 


\section{Introduction}

Industrial Robots are active systems that require a source of energy to power all their functions. The energy needed for operation must be distributed to the various functions and opportunely modulated, by power converters, which are themselves managed by a suitable low level controller. The power converters provide energy to actuators that transduce the electrical energy supplied by the source into the mechanical energy needed to perform the various tasks [1-4]. The actuators provide power to act on the mechanical structure against gravity, inertia, and other external forces to modify the pose of the robot's hand [5].

To choose the components of an actuating system, it is worth starting from the requirements imposed on the mechanical power by the force and velocity that describe the joint motion [6].

The execution of joint motions of a manipulator demands low speeds with high torques [6]. In general, such requirements do not allow an effective use of the mechanical features of electrical motors, which typically provide high speeds with low torques in optimal operating conditions. It is then necessary to interpose a transmission (gear) to optimize the transfer of mechanical power from the motor to the joint. During this transfer, the power is dissipated as a result of friction.

The use of gearing is a well-established means for accomplishing such an objective because it is possible to use smaller actuators to deliver larger torques [7].

However, one of the biggest disadvantages of the addition of gearing is the presence of backlash that reduces the effective bandwidth of the controller since high frequency content in the control produces noisy and destructive operation [8]. Most industrial robot manipulators are driven by motors through gears with high reductions ratios (from tens to a few hundreds). The use of gears permits an optimization of manipulator static and dynamic performance since the motors can be located on the link preceding the actuated joints along the kinematic chain. Further, typical robot applications require motions with large torques and relatively small velocities, and thus the use of gears allows joint actuation by motors of reduced size [8].

The planetary gear system is widely used in robotics $[9,10]$, it is composed of one or more outer gears or planet gears, revolving around a sun gear driven by motor. Typically, the planets are mounted on a movable carrier, which rotates relatively to the sun gear. The planetary gearing systems may also include an outer ring gear or annulus, which meshes with the planet gears [9-11]. A planetary gear example modeled by SolidWorks (2014) is shown in Fig. 1. The advantages of planetary gears over parallel axis gears include high power density, large gear reduction in a small volume, multiple kinematic combinations, pure torsional reactions, and coaxial shafting. Among the disadvantages, one can note high bearing loads, inaccessibility and design complexity $[9,10]$.

The rotary inertia of the sun gear had a very significant influence on the dynamic behavior of planetary gear train [12]. The effect of gear dynamics and gear ratios on the inverse and forward dynamic models is a problem that is of current interest. For the inverse dynamics, the gear dynamics are most commonly approximated by a diagonal matrix added to the mass matrix [13-15]. Spong [16] explicitly assumes that the gyroscopic effects of the spinning rotor and gears are negligible. Springer 
et. $\mathrm{Al}[17]$ and Chen [18] used energy methods to add additional terms to the simple rigid model to account for the effects of gear ratios and gyroscopic forces. Walker and Orin[19] developed methods for the fast calculation of the manipulator mass matrix that could include the common gear approximation.

We model the actuator with planetary gear system by using SolidWorks (2014) with no backlash. The planetary gear serves to deliver high output torques. The sun-gear has as input the torque given by the motor and gives torque to the outer ring gear through planet-gears, the output of the outer ring gear connected with the successive link as shown Fig. 1.

In this paper, we are interested on the effects of the rotary inertia of the number of the equally spaced planet-gears on the dynamic of the robot and we will consider that the planet carrier is fixed. A two-revolute (2-R) planar robot is modeled using the SolidWorks (2014) as shown in Fig. 1. Motors on each joint coupled with a planetary gear system actuate the manipulator that holds an external mass on the end effector.

The rest of this paper is organized as follows: in the section 2 dynamic modeling of the manipulator, section 3 presents the dynamic simulation and in section 4 discussion of the simulation results followed by a conclusion in section 5 .

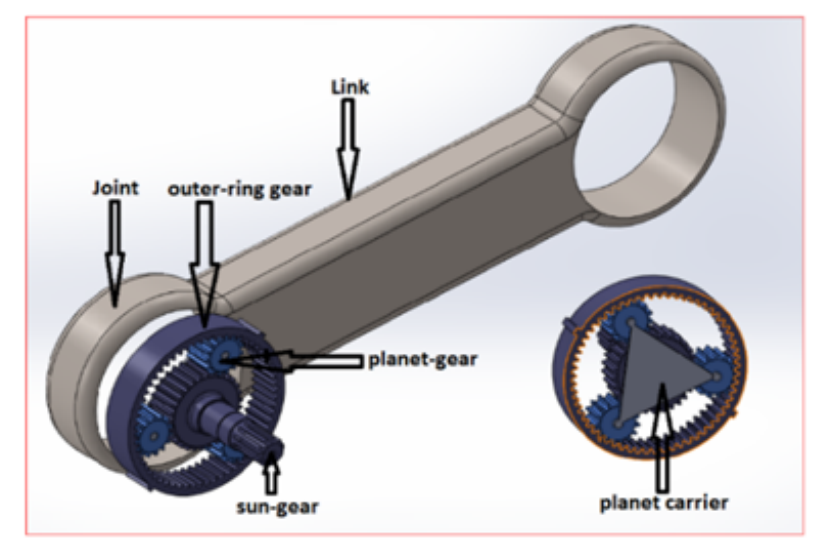

Figure 1 The joint of robot's link actuated by planetary gear system

2. Dynamics modeling of robot actuated by (n) equally spaced planetgears

\subsection{Dynamics modeling of robot actuated by the 3 equally spaced planet-} gears

2.1.1. Computation of Kinetic energy of robot

$$
T=\sum_{i=0}^{n}\left(T_{l_{i}}+T_{s g_{i+1}}+T_{p g 2_{i+1}}+2 T_{p g 1_{i+1}}\right)
$$


It can be assumed that the contribution in the calculation of the kinetic energy of the (ring-gear) is included in that of the link on which the (ring-gear) is located, and thus the sole contribution of the sun-gear and planet-gears are to be computed. The sun-gears are located on the joint axes, the sun-gear (1) and planet-gears (1) are sitting on the ground and their weight will not affect the dynamics of the manipulators $[5,20]$.

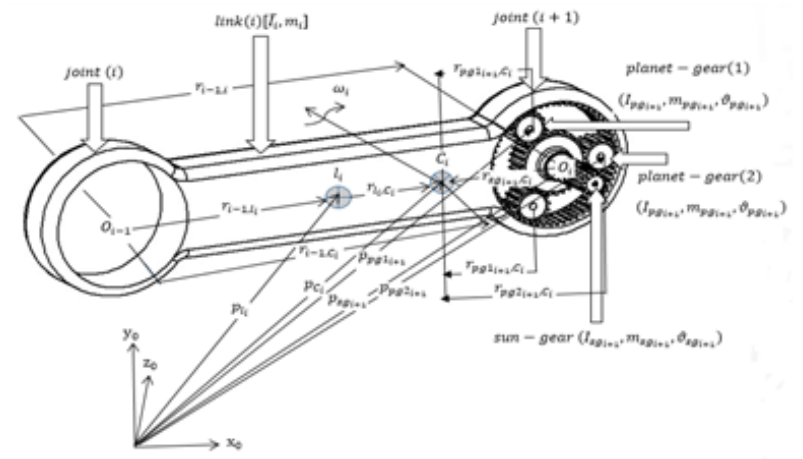

Figure 2 Characterization of augmented link (i) (link+sun-gear+planet-gears) for kinetic energy

The total kinetic energy contribution of Link $(i)$ is given by:

$$
\begin{aligned}
& T=T_{l_{i}}+T_{s g_{i+1}}+T_{p g 2_{i+1}}+2 T_{p g 1_{i+1}}
\end{aligned}
$$

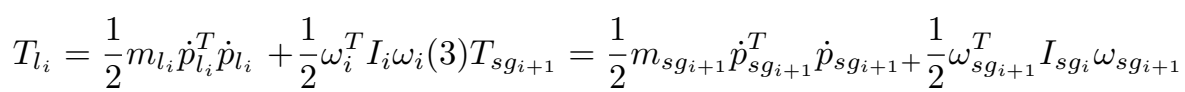

$$
\begin{aligned}
& T_{p g 1_{i+1}}=\frac{1}{2} m_{p g_{i+1}} \dot{p}_{p g_{1 i+1}}^{T} \dot{p}_{p g 1_{i+1}+\frac{1}{2}} \omega_{p g_{i+1}}^{T} I_{p g_{i}} \omega_{p g_{i+1}} \\
& T_{p g 2_{i+1}}=\frac{1}{2} m_{p g_{i+1}} \dot{p}_{p g 2_{i+1}}^{T} \dot{p}_{p g 2_{i+1}}+\frac{1}{2} \omega_{p g_{i+1}}^{T} I_{p g_{i}} \omega_{p g_{i+1}}
\end{aligned}
$$

$\vartheta_{s g_{i+1}}$ and $\vartheta_{p g_{i+1}}$ are the angular position of the sun-gear and planet-gear respectively.

$$
\begin{aligned}
& \dot{\vartheta}_{s g_{i+1}}=G_{s g r_{i+1}} \dot{\vartheta}_{i} \\
& \dot{\vartheta}_{p g_{i+1}}=G_{p g r_{i+1}} \dot{\vartheta}_{i}
\end{aligned}
$$

here $G_{s g r_{i+1}}$ and $G_{p g r_{i+1}}$ are the gear reduction ratios.

In our case, the planet carrier is fixed, the gear reduction ratios can be calculated by using the relation of Willis [24-26]:

$$
\begin{gathered}
G_{s g r_{i+1}}=G_{s g r_{i}}=\frac{Z_{r g}}{Z_{s g}}=\frac{\vartheta_{s g_{i}}}{\vartheta_{i}} \\
G_{p g r_{i+1}}=G_{p g r_{i}}=\frac{Z_{r g}}{Z_{p g}}=\frac{\vartheta_{p g_{i}}}{\vartheta_{i}}
\end{gathered}
$$


Where $Z_{s g}$ the number of sun-gear teeth is, $Z_{r g}$ is the number of ring-gear teeth, and $Z_{p g}$ is the number of planet-gear teeth. The ring-gear rotates at the rate $G_{s g r_{i+1}}$ in the negative direction of the sun-gear because the sun gear and the ring gear rotate through a planet gear.

In similar fashion, the kinetic energy of the augmented link (Link+sun-gear+planetgears) actuated by ( $n$ ) equally planet-gears:

$$
\begin{aligned}
& T_{i}=\frac{1}{2} m_{i} \dot{p}_{i}^{i^{T}} \dot{p}_{i}^{i}+\dot{p}_{i}^{i^{T}} S\left(\omega_{i}^{i}\right) m_{i} r_{i, C_{i}}^{i} \\
& +\frac{1}{2} \omega_{i}^{i^{T}} \overline{\bar{I}}_{i}^{i} \omega_{i}^{i}+G_{s g r, i+1} \dot{q}_{i+1} I_{s g_{i+1}} z_{s g_{i+1} i^{T}} \omega_{i}+\frac{1}{2} G_{s g r, i+1}^{2} \dot{q}_{i+1}^{2} I_{s g_{i+1}} \\
& +(n) G_{p g r, i+1} \dot{q}_{i+1} I_{p g_{i+1}} z_{p g_{i+1}}^{i^{T}} \omega_{i}+\frac{(n)}{2} G_{p g r}^{2}, i+1 \dot{q}_{i+1}^{2} I_{p g_{i+1}}
\end{aligned}
$$

\subsubsection{Newton-Euler Formulation}

The Newton-Euler formulation describes the motion of the link in terms of a balance of forces and moments acting on it [21, 22], as shown in Fig 3.

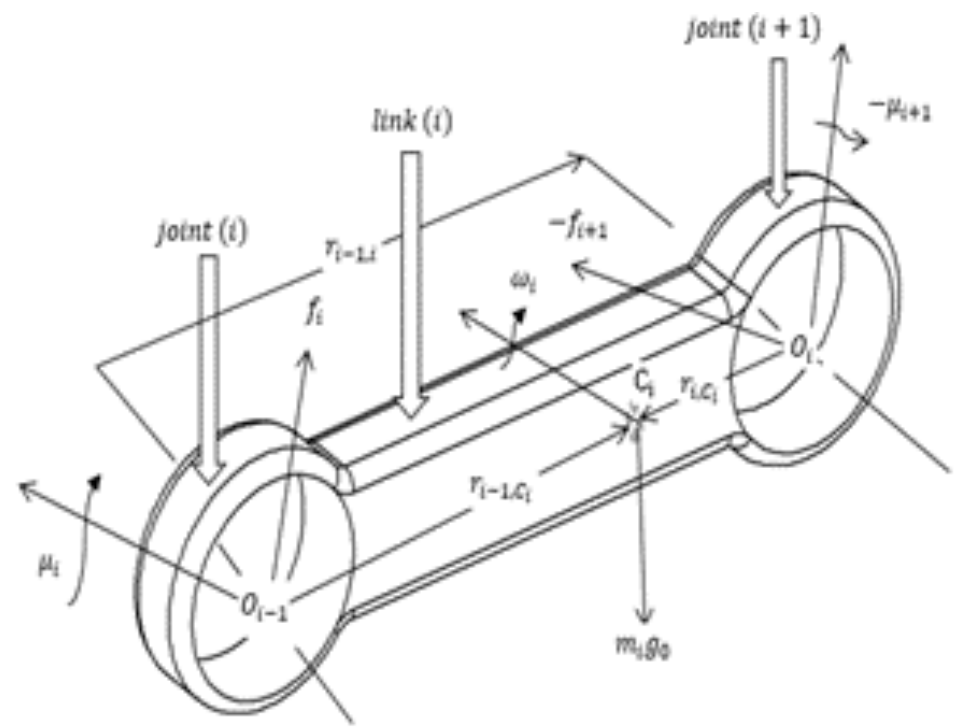

Figure 3 Characterization of augmented link $(i)$ for Newton-Euler formulation

\subsubsection{The joint exerted at the joint (i) in current frame}

The equation of the joint torque of robot's link actuated by 3 equally spaced planetgear:

$$
\tau_{i}^{i}=\mu_{i}^{i^{T}} R_{i}^{i-1^{T}} z_{0}+G_{s g r, i} I_{s g_{i}} \dot{\omega}_{s g_{i}}^{i-1^{T}} z_{s g_{i}}^{i-1}+3 G_{p g r, i} I_{p g_{i}} \dot{\omega}_{p g_{i}}^{i-1^{T}} z_{p g_{i}}^{i-1}
$$


In similar fashion, the equation of the joint torque of robot's link actuated by ( $n$ ) equally planet-gears:

$$
\begin{aligned}
& \tau_{i}^{i}=\mu_{i}^{i^{T}} R_{i}^{i-1^{T}} z_{0}+G_{s g r, i} I_{s g_{i}} \dot{\omega}_{s g_{i}}^{i-1^{T}} z_{s g_{i}}^{i-1}+(n) G_{p g r, i} I_{p g_{i}} \dot{\omega}_{p g_{i}}^{i-1^{T}} z_{p g_{i}}^{i-1} \\
& \mu_{i}^{i}=-f_{i}^{i} \times\left(r_{i-1, i}^{i}+r_{i, C_{i}}^{i}\right)+R_{i+1}^{i} \mu_{i+1}^{i+1}+R_{i+1}^{i} f_{i+1}^{i+1} \times r_{i, C_{i}}^{i}+\overline{\bar{I}}_{i}^{i} \dot{\omega}_{i}^{i}+ \\
& \omega_{i}^{i} \times\left(\overline{\bar{I}}_{i}^{i} \omega_{i}^{i}\right)+G_{s g r, i+1}\left(\underset{i+1}{q} I_{s g_{i+1}} z_{s g_{i+1}}^{i}+\dot{q}_{i+1} I_{s g_{i+1}} \omega_{i}^{i} \times z_{s g_{i+1}}^{i}\right)+ \\
& (n) G_{p g r, i+1}\left(\underset{i+1}{q} I_{p g_{i+1}} z_{p g_{i+1}}^{i}+\dot{q}_{i+1} I_{p g_{i+1}} \omega_{i}^{i} \times z_{p g_{i+1}}^{i}\right)
\end{aligned}
$$

Table 1 The mass properties of 2-R robot actuated by (n) equally spaced planet-gears by using SolidWorks

\begin{tabular}{|l|l|l|}
\hline Parameter & Value & Unit \\
\hline Mass of augmented link $(1)$ actuated by 3 ESPG $\left(m_{i}\right)$ & 29 & $\mathrm{Kg}$ \\
\hline Mass of augmented link(1) actuated by 4 ESPG $\left(m_{i}\right)$ & 29.12 & $\mathrm{Kg}$ \\
\hline $\begin{array}{l}\text { The inertia tensor of the augmented link }(1) \text { actuated by } 3 \\
\text { ESPG }\left(\overline{\overline{I_{1 z z}^{1}}}\right)\end{array}$ & 0.40 & $\mathrm{Kgm}^{2}$ \\
\hline $\begin{array}{l}\text { The inertia tensor of the augmented link }(1) \text { actuated by } 4 \\
\text { ESPG }\left(\overline{\overline{I_{1 z z}^{1}}}\right)\end{array}$ & 0.4028 & $\mathrm{Kgm}^{2}$ \\
\hline The mass of the augmented link $(2)($ link+external mass $)$ & 29 & $\mathrm{Kg}$ \\
\hline $\begin{array}{l}\text { The inertia tensor of the augmented link}(2)(\text { link+external } \\
\text { mass })\left(\overline{\overline{I_{2 o z z}^{2 o}}}\right)\end{array}$ & 0.43 & $\mathrm{Kgm}^{2}$ \\
\hline The inertia tensor of the sun-gear $\left(I_{s g_{i+1(z z)}}\right)$ & 0.01574 & $\mathrm{Kgm}^{2}$ \\
\hline The inertia tensor of the planet-gear $\left(I_{\left.p g_{i+1(z z)}\right)}\right)$ & 0.00314 & $\mathrm{Kgm}^{2}$ \\
\hline $\begin{array}{l}\text { The gear reduction ratios between the sun-gear and ring-gear } \\
\left(G_{s g r, i+1}=G_{s g r, i}\right)\end{array}$ & 2 & \\
\hline $\begin{array}{l}\text { The gear reduction ratios between the planet-gear and ring- } \\
\text { gear }\left(G_{p g r, i+1}=G_{p g r, i}\right)\end{array}$ & 4 & \\
\hline
\end{tabular}

\section{Dynamic simulation}

As an example of simulation, we choose an elbow down of 2-R planar robot. The links of robot have the same length $(0.4 \mathrm{~m})$ and actuated by $3,4,5$ and 6 equally spaced planet-gears. The simulation of the kinetic energy and the joint torque can be done by using the mass properties shown in Table 1. The links of robot, sungear, planet-gears and external mass are designed by using SolidWorks (2014) in such way the inertia tensor of link, sun-gear, planet-gears and external mass has a diagonal matrix about its center of mass in current frame, then the inertia tensor of the augmented link is also diagonal matrix about its overall center of mass $C_{i}$ in current frame.

The 2-R robot moves between two positions during $1 \mathrm{~s}(\mathrm{x}=0.8 \mathrm{~m}, \mathrm{y}=0 \mathrm{~m})$ to $(\mathrm{x}=0.4499 \mathrm{~m}, \mathrm{y}=0.5864 \mathrm{~m})$ with jerk zero at start-stop path. Generation of trajectories with a bounded value of jerk improves the tracking accuracy and will 
allow to reach a higher speed of task execution, with eventually a reduction in the excitation of the resonant frequencies and the vibrations caused by the planetary gear system [23-26]. We can compare between the two postures of 2-R robot by using the equations of the serial planar manipulators [27] in the dynamics modeling shown in section 2 to choose the posture that has the power saving [28-34].

\subsection{Kinetic energy simulation}

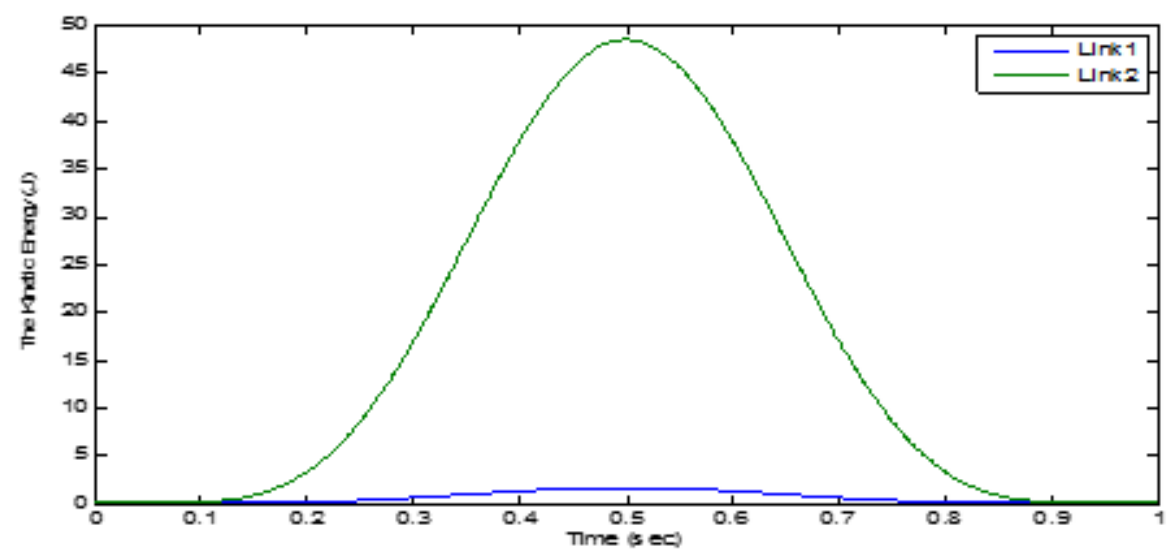

Figure 4 The kinetic energy of robot actuated by 3 ESPG

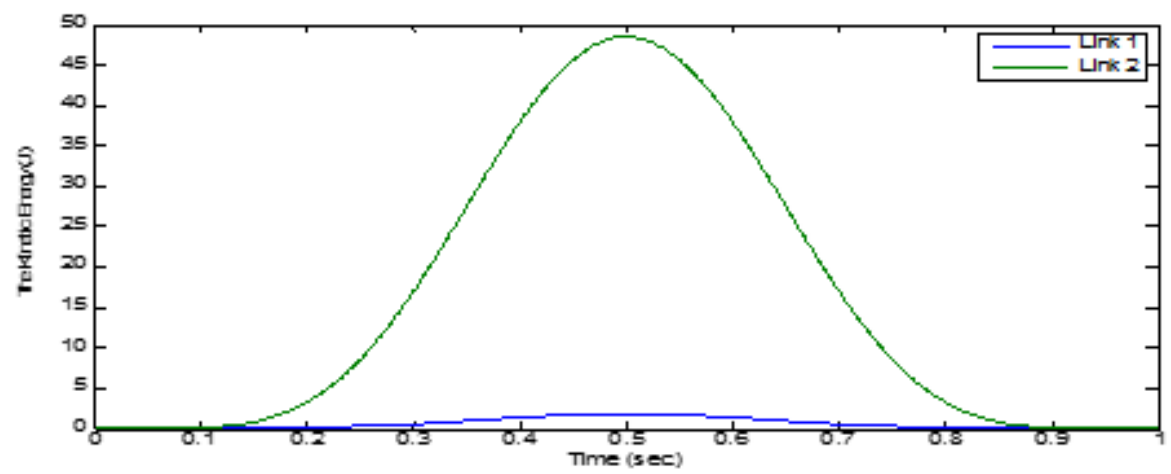

Figure 5 The kinetic energy of robot actuated by 4 ESPG 


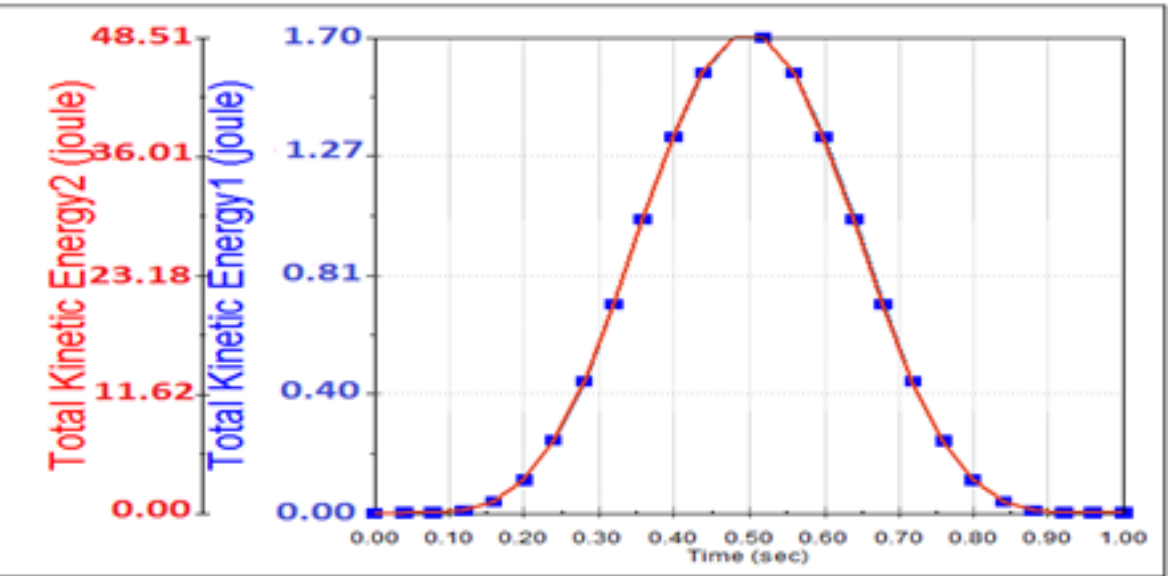

Figure 6 The kinetic energy of robot actuated by 3 ESPG by using SolidWorks (2014)

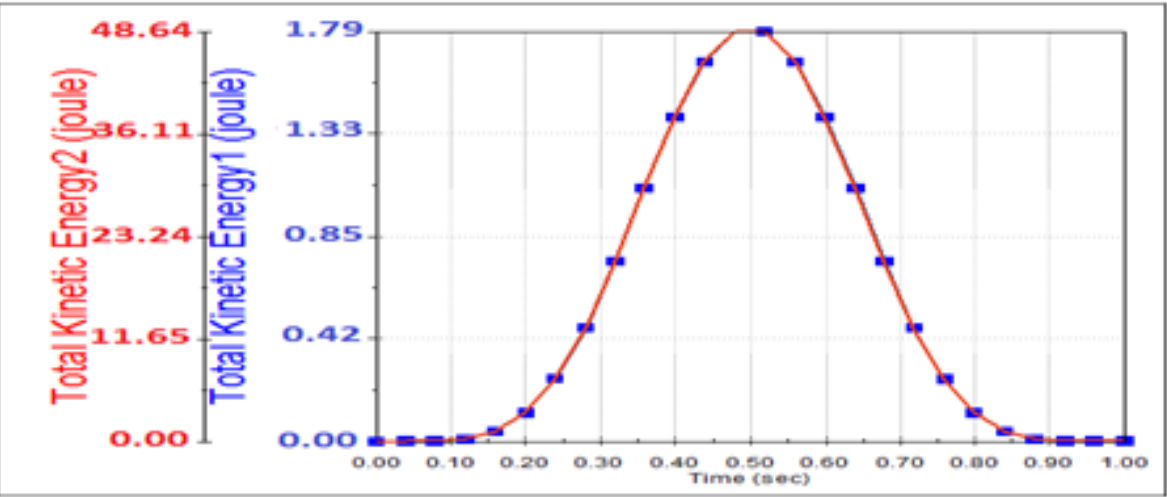

Figure 7 The kinetic energy of robot actuated by 4 ESPG by using SolidWorks (2014) 


\subsection{Joint torque simulation}

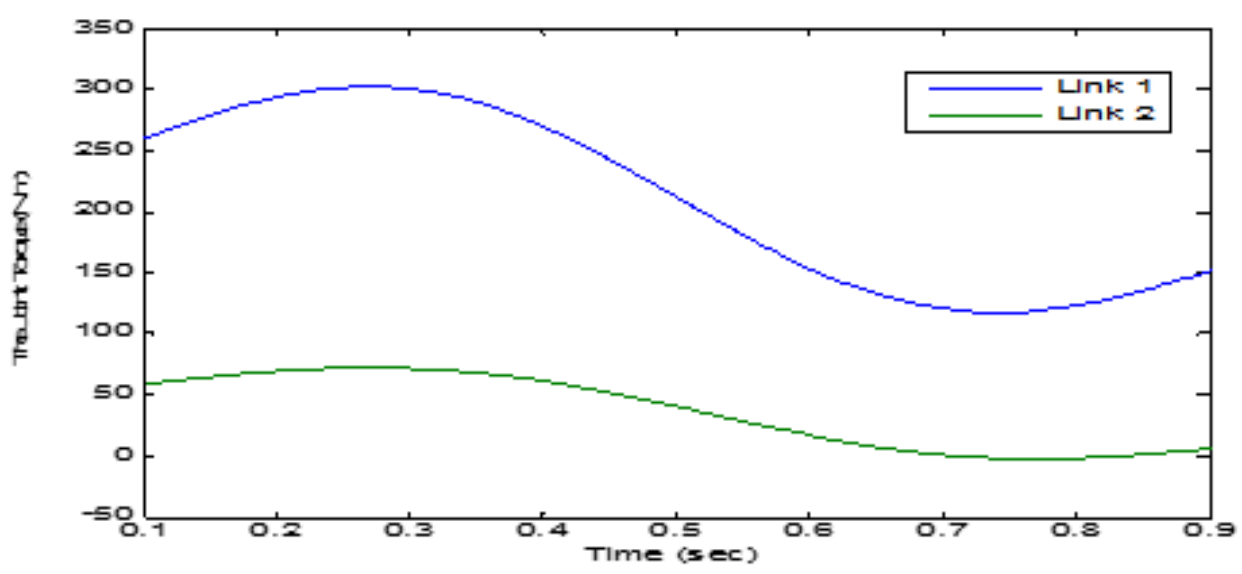

Figure 8 The variation of joint torques of links of robot actuated by 3 ESPG

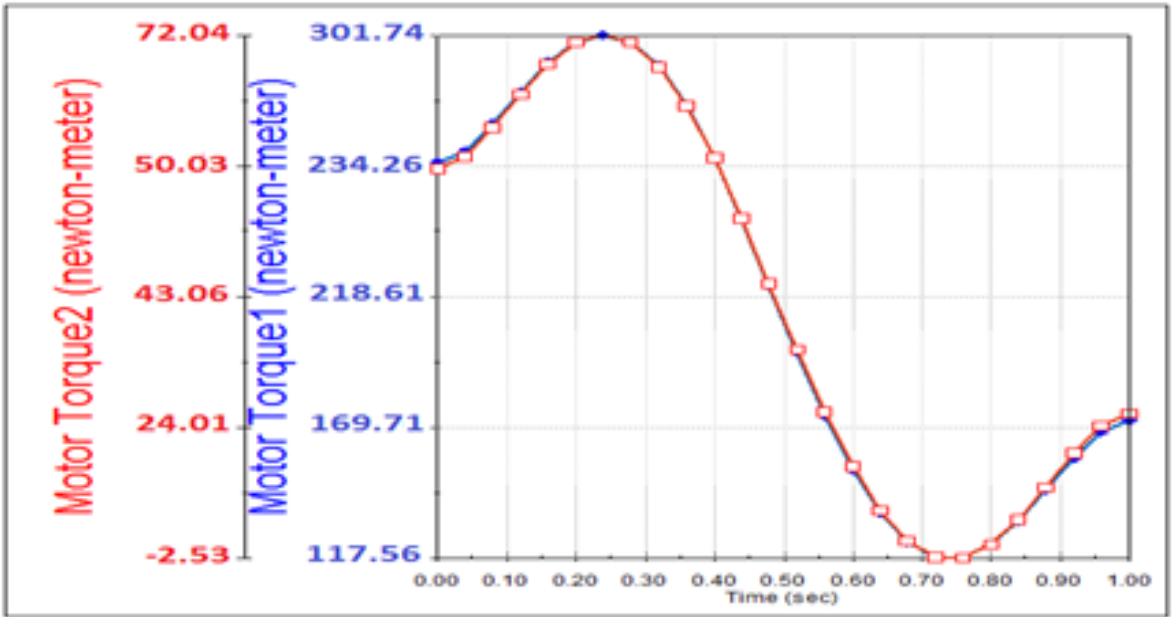

Figure 9 The variation of joint torques of links of robot actuated by 3 ESPG by using SolidWorks (2014) 


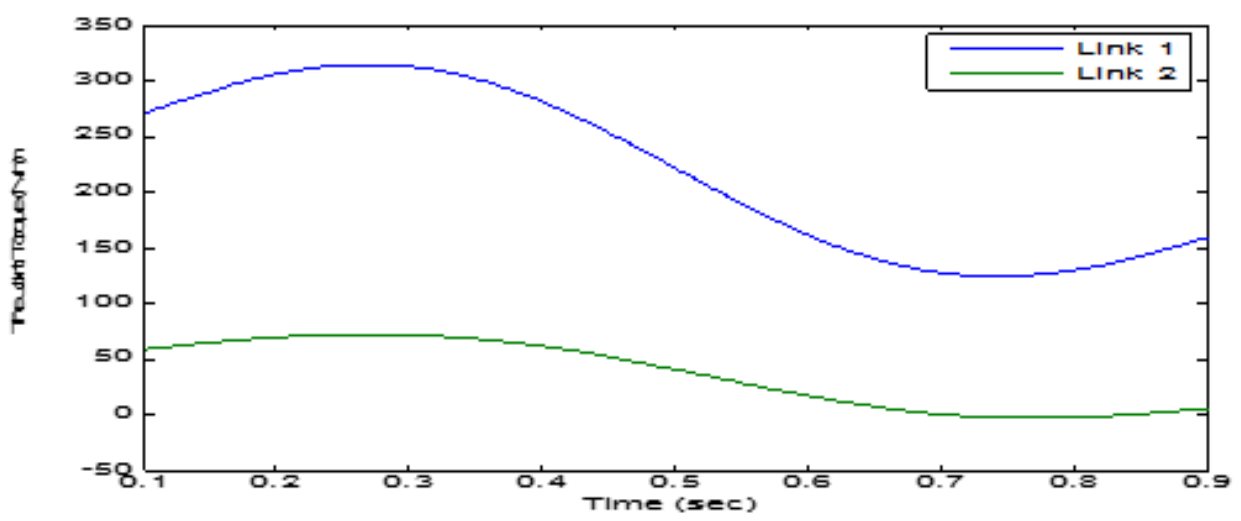

Figure 10 The variation of joint torques of links of robot actuated by $4 \mathrm{ESPG}$

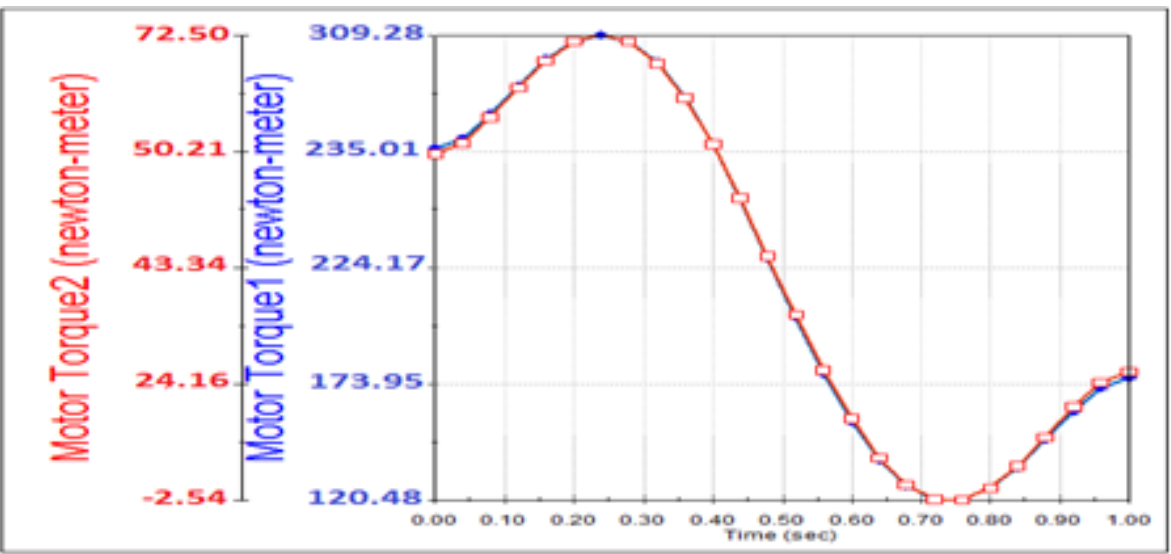

Figure 11 The variation of joint torques of links of robot actuated by 4 ESPG by using SolidWorks (2014)

\section{Discussion}

We can notice from the results obtained from the dynamic simulation of a 2-R robot shown in section 3 that the results obtained whether using simulation results or SolidWorks (2014) are the same as shown in the Figures from 4 to 11. This similarity of results confirms the reliability and correctness of the dynamic model studied. We can also notice from this study that, with the presence of the rotary inertia of the number of the equally planet gears,

(n) $G_{p g r, i+1} \dot{q}_{i+1} I_{p g_{i+1}} z_{p g_{i+1}}^{i^{T}} \omega_{i}+\frac{(n)}{2} G_{p g r}^{2}, i+1 \dot{q}_{i+1}^{2} I_{p g_{i+1}}$ in the equation of the kinetic 
energy of $\operatorname{link}(1) \operatorname{and}(n) G_{p g r, i+1}\left(\underset{i+1}{q} I_{p g_{i+1}} z_{p g_{i+1}}^{i}+\dot{q}_{i+1} I_{p g_{i+1}} \omega_{i}^{i} \times z_{p g_{i+1}}^{i}\right)$ in the equation of the joint torque of link (1), the value of ratio between the kinetic energy and the joint torque actuated by $(n+1)$ and $(n)$ ESPG is $\mathbf{1 . 0 5}$ and $\mathbf{1 . 0 2 5}$ respectively, this fact is clear from the Figures from 4 to11 (only the simulation of the kinetic energy and the joint torque of the links actuated by 3 and 4 ESPG is shown in this paper, and the simulation results are verified also by the links which is actuated by 5 and 6 ESPG). Another fact is revealed from these Figures is that, the absence of the rotary inertia of the number of the ESPG in the equation of kinetic energy and in the equation of the joint torque of link (2), the values of the kinetic energy and the joint torque actuated by $(n+1)$ and $(n)$ equally spaced planet-gears are almost the same.

\section{Conclusions}

The dynamics modeling of robot actuated by $(n)$ equally spaced planet-gears are studied. The verification of the dynamic modeling of a 2-R robot actuated by $(n)$ equally spaced planet-gears by using the software SolidWorks (2014) permitted us to qualitatively develop and highlight the relevance of the dynamic model studied. From this research The authors found that the effect of the rotary inertia of the number of the ESPG on the dynamic of 2-R robot with jerk zero at start-stop path is the augmentation of the kinetic energy and the joint torque of the robot's link actuated by $(n+1)$ ESPG by a percentage of $\mathbf{5 \%}$ and $\mathbf{2 . 5 \%}$ respectively, relative to the Kinetic energy and the joint torques of the robot's link actuated by $(n)$ equally spaced planet-gears for a same trajectory planning.

\section{References}

[1] Giancarlo, G.: Actuators and Sensors Introduction to the Mechanics of Space Robots, Space Technology Library, Volume26 Springer, 427-482, 2012.

[2] Oriol, G., Lucio, F.: Actuator Principles and Classification Design Rules for Actuators in Active Mechanical Systems, Springer, 3-28, 2010.

[3] Gomis, O.: Design, modeling, identification and control of mechatronic systems, $\mathrm{PhD}$ thesis, Technical University of Catalonia, 2007.

[4] Gomis, O., Galceran, S., Sudri‘a, A., Montesinos, D.: Linear electromagnetic actuator modeling for optimization of mechatronic and adaptronic systems, Campanile LF, 2007.

[5] Reza, N.: Theory of Applied Robotics Kinematics, Dynamics, and Control, 2nd Edition Springer, 2010.

[6] Bruno, S., Lorenzo, S., Giuseppe, O.: Robotics Modelling, Planning and Control, Springer, 2009.

[7] Bridges, M., Dawson, D.: Redesign of robust controllers for rigid link flexible joint robotic manipulators actuated with harmonic drive gearing, IEE Proceedings Control Theory and Applocations, 142, 5, September, 1995.

[8] Bruno, S., Lorenzo, S., Luigi, V.: Lagrange and Newton-Euler dynamic modeling of a gear-driven robot manipulator with inclusion of motor inertia effects, Advanced Robotics, 1995. 
[9] Gyula, M., Szilvester, P., Gizella, P., Zolth, J.: Flexible Planetary Gear Drives In Robotics,IEEE International Conference on Industrial Electronics, Control, Instrumentation ,Automation, Power Electronics and Motion Control, 1992.

[10] Koichi, K., Akira, I.: Artificial Hand Based on the Planetary Gear SystemRealization of Daily Utility Motion of a Hand with Minimum Actuators, IEEE International Conference on Mechatronics and Automation ICMA, 2013.

[11] Hoyul, L., Youngjin, C.: A New Actuator System Using Dual-Motors and a Planetary Gear, IEEE/ASME Transactions Mechatronics, 2012.

[12] Yan, C., Xin, W., Jing J.: Influence of the Sun Gear on the Dynamical Behavior of Planetary Gear Train, IEEE International Conference on Intelligent Human-Machine Systems and Cybernetics, 2009.

[13] Murphy, S., Wen, J., Saridis, G.: Recursive calculation of geared robot manipulator dynamics, IEEE Proceedings International Conference on Robotics and Automation, 1990.

[14] Paul, R.: Mathematics, Programming, and Control, Robot Manipulators The MIT Press, Cambridge Mass, 1981.

[15] Asada, H., Slotine, H.: Robot Analysis and Control, John Wiley and Sons, 1986.

[16] Spong, M.: Modeling and Control of Elastic Joint Robots, Journal of Dynamic Systems, Measurement, and Control, 1987.

[17] Lugner, A., Desoyer, K.: Equations of Motion of Manipulators Including Dynamic Effects of Active Elements, IFAC Symposium on Robot Control (SYROC0'85), 1985.

[18] Chen, J.: The Effects of Gear Reduction on Robot Dynamics, Proceedings of the NASA Conference on Space Tele-robotics, 1989.

[19] Walker, M., Orin, D.: Efficient Dynamic Computer Simulation of Robotic Mechanisms. Journal of Dynamic Systems, Measurement, and Control, 1982.

[20] John, J.: Introduction to robotics Mechanics and control. Third edition, 2005.

[21] Corke, P.: A robotics toolbox for MATLAB, IEEE Robotics \& Automation Magazine 1996.

[22] Sisir, K.: On the dynamics of SCARA robot, Robotics and Autonomous Systems, 1992.

[23] Gasparetto,A., Lanzutti,A., Vidoni,R., Zanotto,V.: Experimental validation and comparative analysis of optimal time-jerk algorithms for trajectory planning, Robotics and Computer-Integrated Manufacturing, 2012.

[24] Barre, P., Bearee, R., Borne, P., Dumetz, E.: Influence of a jerk controlled movement lawon the vibratory behaviour of high-dynamics systems, Journal of Intelligent and Robotic Systems, 2005.

[25] Sonja, M., Elizabeth, A.: Jerk Bounded Manipulator Trajectory Planning Design for Real Time Applications, IEEE Transactions On Robotics And Automation, 2003.

[26] Piazzi, A., Visioli, A.: Global minimum-jerk trajectory planning of robot manipulators. IEEE Transactions on Industrial Electronics, 2000.

[27] Fernini, B., Temmar, M.: An algorithm for serial planar manipulators that have an architecture R// (n) R by using Solidworks (2013) and Matlab/Simulink (2012), IEEE International Confrence on Mechatronics and Automation ICMA, 2013.

[28] Gouasmi, M., Ouali, M., Fernini, B., Meghatria, M.: Kinematic modeling and simulation of a 2-R robot using Solidworks and verification by Matlab/Simulink, International Journal of Advanced Robotic Systems, 2012.

[29] Fernini, B., Temmar, M.: Dynamic Behavior of a SCARA Robot by using NE Method for a Straight Line and Simulation of Motion by using Solidworks and 
Verification by Matlab/Simulink, International Journal of Robotics and Automation, 2014.

[30] Fernini, B., Temmar, M.: The Effect of Mono and Biarticular Muscles on the Dynamic of Waliking Bipedal Robot, IEEE INTELLISYS, 2017.

[31] Fernini, B., Temmar, M., Noor, MM.: Toward a Dynamic Analysis of Bipedal Robots Inspired By Human Leg Muscles, Journal of Mechanical Engineering and Sciences, 2018.

[32] Fernini, B., Temmar, M.: The Positions Effect of Biarticular Muscles on the Walking Fatigue of Bipedal Robots, Receuil De Mecanique, 2016.

[33] Gouasmi, M., Ouali, M., Fernini, B.: Robot Kinematics Using Dual Quaternions, International Journal of Robotics and Automation, 2012.

[34] Fernini, B.: Kinematic Modeling and Simulation of a 2-R Robot Using Solidworks and Verification by Matlab/Simulink, International Journal of Robotics and Automation, 2012.

\section{Nomenclature:}

$C_{i}$ : Center of mass of augmented link.

$\overline{\bar{I}}_{i}$ : Inertia tensor of augmented link (link+sun-gear+ planet-gears).

$\overline{\bar{I}}_{i 0}$ : Inertia tensor of augmented link (link+external mass).

$I_{s g_{i+1}}$ : Moment of inertia of sun-gear about its center of mass.

$I_{p g_{i+1}}$ : Moment of inertia of planet-gear about its center of mass.

$r_{i, C_{i}}$ : Vector from origin of frame $(i)$ to center of mass $C_{i}$.

$r_{i-1, l_{i}}$ : The vector from origin of frame $(i-1)$ to center of mass of link $(i)$.

$r_{C_{i}, s g_{i+1}}$ : The vector from the center of mass $C_{i}$ to the joint axis where the sun-gear is located.

$r_{C_{i}, e_{m}}$ : The vector from the center of mass $C_{i}$ to center of mass of external mass.

$\ddot{P}_{C_{i}}$ : Linear acceleration of center of mass $C_{i}$.

$\ddot{P}_{i}$ : Linear acceleration of origin of frame $(i)$.

$\dot{\omega}_{i}$ : Angular acceleration of link $(i)$.

$\omega_{p g_{i}}$ : Angular velocity of planet-gear.

$\dot{\omega}_{p g_{i}}$ : Angular acceleration of planet-gear.

$m_{l_{i}}$ : Mass of link $(i)$.

$I_{l_{i}}$ : Moment of inertia of link $(i)$.

$m_{s g_{i+1}}$ : Mass of sun-gear.

$m_{p g_{i+1}}$ : Mass of planet-gears.

$f_{i}$ : Force exterted by link $(i-1)$ on link $(i)$.

$P_{s g_{i+1}}$ : Vector from base coordinate system $\left(\mathrm{x}_{0}, \mathrm{y}_{0}, \mathrm{z}_{0}\right)$ to the joint axis where the sun-gear is located.

$\dot{p}_{s g_{i+1}}$ : Linear velocity from base coordinate system $\left(\mathrm{x}_{0}, \mathrm{y}_{0}, \mathrm{z}_{0}\right)$ to the joint axis where the sun-gear is located.

$\mathrm{Z}_{r g}$ : The number of ring gear teeth.

$\mathrm{Z}_{p g}$ : The number of planet-gear teeth.

$R_{i}$ : The rotation matrix from Frame $(i)$ to base frame $\left(\mathrm{x}_{0}, \mathrm{y}_{0}, \mathrm{z}_{0}\right)$.

$T_{l_{i}}$ : The kinetic energy of link $(i)$.

$m_{\mathrm{i}}$ : Mass of augmented link (link+sun-gear+planet-gears).

$r_{i-1, C_{i}}$ : Vector from origin of frame $(i-1)$ to center of mass $C_{i}$.

$r_{i-1, i}$ : Vector from origin of frame $(i-1)$ to the frame $(i)$. 
$r_{C_{i}, l_{i}}$ : The vector from the center of mass $C_{i}$ to center of mass of link $(i)$.

$r_{C_{i}, p g_{i+1}}$ : The vector from the center of mass $C_{i}$ to the center of mass of the planet-gear.

$\dot{P}_{C_{i}}$ : Linear velocity of center of mass $C_{i}$.

$\dot{P}_{i}$ : Linear velocity of origin of frame $(i)$.

$\omega_{i}$ : Angular velocity of link $(i)$.

$\omega_{s g_{i}}$ : Angular velocity of sun-gear.

$\dot{\omega}_{s g_{i}}$ : Angular acceleration of sun-gear.

$g_{0}$ : Gravity accelaration.

$-f_{i+1}$ : Force exterted by link $(i+1)$ on link $(i)$.

$\mu_{i}$ : Moment exterted by link $(i-1)$ on link $(i)$ with respect to origin of frame $(i-1)$.

$P_{C_{i}}$ : Vector from base coordinate system $\left(\mathrm{x}_{0}, \mathrm{y}_{0}, \mathrm{z}_{0}\right)$ to position of center of mass of augmented link $(i)$.

$S$ : Skew symetric matrix.

$\mathrm{Z}_{s g}$ : The number of sun-gear teeth. (i)

$\mu_{i+1}$ : Moment exerted by link $(i+1)$ on link $(i)$ with respect to origin of frame

$\dot{p}_{l_{i}}$ : Linear velocity from base coordinate system $\left(\mathrm{x}_{0}, \mathrm{y}_{0}, \mathrm{z}_{0}\right)$ to center of mass of link $(i)$.

$P_{p g_{i+1}}$ : Vector from base coordinate system $\left(\mathrm{x}_{0}, \mathrm{y}_{0}, \mathrm{z}_{0}\right)$ to the center of mass of planet-gear.

$G_{s g r_{i+1}}:$ The gear reduction ratios between sun-gear and ring-gear.

$G_{p g r_{i+1}}$ :The gear reduction ratios between planet-gear and ring-gear.

$T_{s g_{i+1}}$ : The kinetic energy of the sun-gear.

$T_{i}$ : The Kinetic energy of the augmented link.

$T_{p g_{i+1}}$ : The kinetic energy of the planet-gear.

$\dot{p}_{p g_{i+1}}$ : Linear velocity from base coordinate system $\left(\mathrm{x}_{0}, \mathrm{y}_{0}, \mathrm{z}_{0}\right)$ to the center of mass of planet-gear.

Abbreviation

ESPG: Equally spaced planet-gears. 